\title{
Color-Coding Point Group and Space Group Diagrams
}

\author{
Carla Slebodnick ${ }^{\mathrm{a}}$ and Maureen M. Julian ${ }^{\mathrm{b}}$
}

Department of Chemistry, Virginia Tech, Blacksburg, Virginia 24061, slebod@vt.edu Department of Materials Science and Engineering, Virginia Tech, Blacksburg, Virginia, 24061, erie@vt.edu.

Color-coding according to proper vs. improper symmetry operations deepens the understanding of the point group and space group diagrams because change of handedness is an intrinsic property of symmetry operations. The purpose of this paper is to emphasize this relationship in the space group diagrams. The symmetry operations are coded red if there is no change of handedness and blue if there is a change. The graphical symbols for the symmetry elements are coded red for the rotation and screw axes because they are associated with only proper symmetry operations. The symbols for the rotoinversion axes and glide planes are coded purple because the group contains an equal numbers of red and blue symmetry operations. On the other hand, when the inversion point is combined with an even rotation axis, then two colors are needed; the inversion point is purple and the even rotation axis is red. For example, in Figure 1 the symbol at the origin is the red $6_{3}$ screw axis with the superimposed purple inversion point.
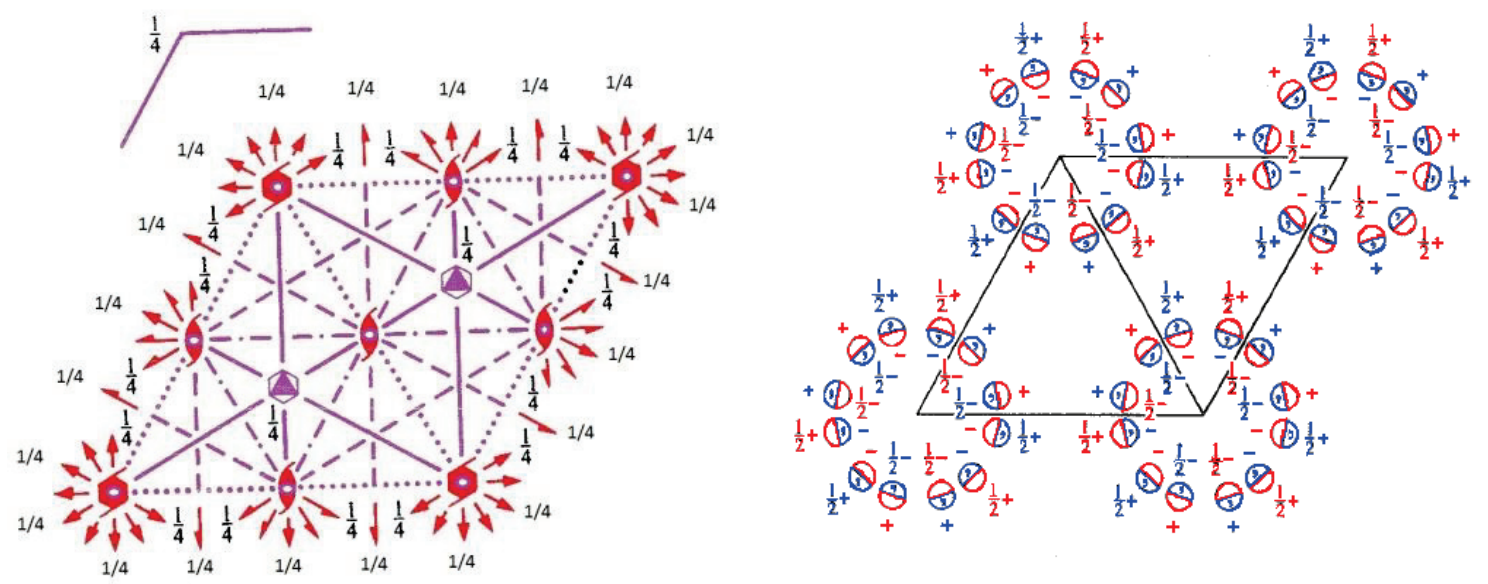

Figure 1. Color-coded space group diagrams for $P 6_{3} / m m c$.[1]

References

[1] Julian, M. M. Foundations of Crystallography with Computer Applications, $2^{\text {nd }}$ edition, CRC Press: New York (2015), ISBN: 978-1466552913. Figures 8.2b and 8.4b. 